\title{
Einbecker Empfehlungen der DGMR zu Rechtsfragen der Personalisierten Medizin
}

Was verbirgt sich hinter dem Schlagwort „Personalisierte Medizin“? Ist es die besondere $\mathrm{Zu}$ wendung des Arztes zu seinem Patienten oder das Eingehen auf die persönliche, einzigartige Lebenssituation des Kranken? Ist es die Implantation einer maßgefertigten Endoprothese als Unikat oder die Behandlung mit aus Stammzellen generierten Koronararterien? Oder dann doch „nur“ die Zuordnung der Patienten zu „Strata“, zu Kategorien oder Subpopulationen, die für definierte Therapien geeignet sind oder nicht? Personalisierte Medizin ist nicht mit einer exakten Definition belegt und die Diskussion um sie läuft stets Gefahr, sich in einer Diskussion um Begrifflichkeiten zu verlieren. Die Deutsche Gesellschaft für Medizinrecht, DGMR, hat sich im Jahr 2013 gerade deshalb dem Thema verschrieben und legt mit diesem Tagungsband nicht nur die Referate zum Workshop, sondern auch erwartungsgemäß die „Einbecker Empfehlungen“ vor, die als Ergebnis dieser Tagung unter Abstimmung aller Beteiligten entstanden sind.

Aus dem weiten Spektrum eines potenziellen Verständnisses „personalisierter Medizin“ haben sich die teilnehmenden Experten der Medizin, der Rechtswissenschaft und der Versicherungswirtschaft auf die Analyse und Bewertung eines Teilbereichs verständigt, der auch als „molekulare Medizin“ bezeichnet werden kann, da er im Wesentlichen die Diagnostik und therapeutische Beeinflussung molekularer Mechanismen der Krankheit in den
Fokus nimmt. Neu ist bei dieser Therapie nicht der Ansatz an den molekularen Mechanismen der Krankheit, sondern die durch molekulare, zum Teil auch genetische Diagnostik entstandenen Möglichkeiten der Vorhersage von Arzneimittelwirkungen. Seit den 50er Jahren ist bekannt, dass der Arzneimittelstoffwechsel genetischer Determinierung unterworfen ist. Die Metabolisierung von Wirkstoffen und ihrer Vorstufen (prodrugs) ist ausschlaggebend für die Wirkung. Manche Arzneimittel müssen durch die körpereigenen Enzyme erst in die aktiven Metaboliten überführt werden, alle werden durch den Arzneimittelstoffwechsel abgebaut und ausgeschieden. Der therapeutische Effekt als area under the curve hängt von der interindividuell unterschiedlichen Enzymausstattung, z.B. der Cytochrome ab. Andere Arzneimittel setzen an definierten Rezeptormolekülen von Tumoren an oder greifen gezielt in die Signalwege des Tumorwachstums ein (target molecules). Das erweiterte Verständnis der Pathomechanismen und die daraus resultierenden Möglichkeiten einer targeted therapy prägen den Begriff der „molekularen Medizin“.

Wann ist die Diagnostik von Biomarkern Standard? Wie verhält es sich mit der Einstandspflicht der Gesetzlichen Krankenversicherung für eine Pharmakotherapie, wenn die notwendige Bestimmung des Biomarkers noch nicht abrechenbar ist? Welche Auswirkungen haben falsch negative Biomarker-Bestimmungen auf

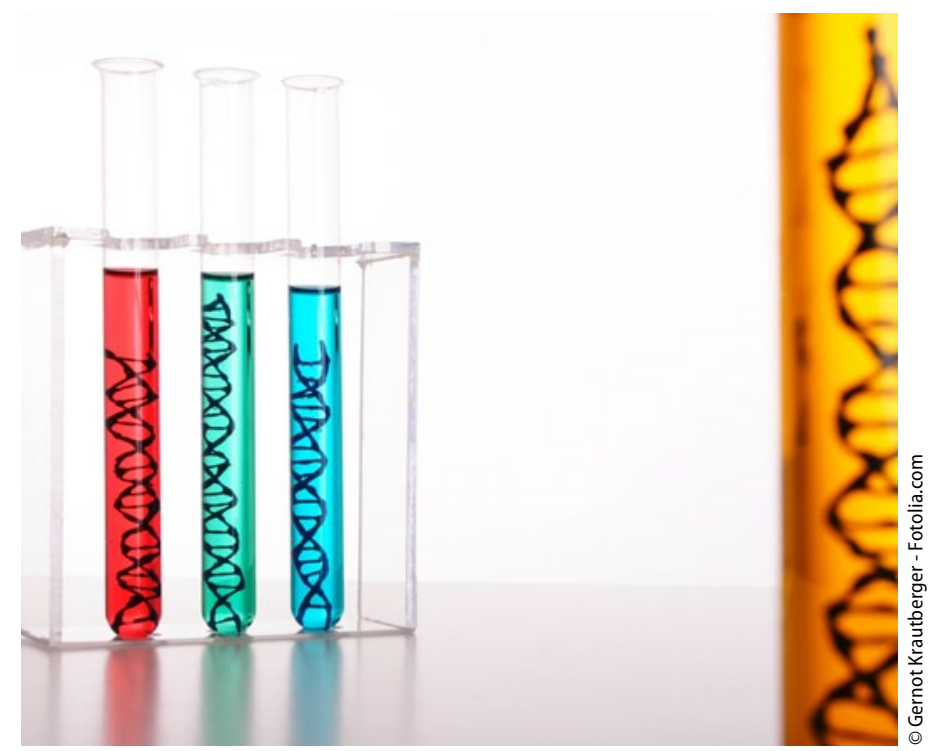

A Die „personalisierte Medizin“ eröffnet durch molekulare und genetische Diagnostik neue Vorhersagemöglichkeiten über Wirkung von Arzneimitteln.

den Anspruch des Patienten? Der Einbecker Workshop beleuchtet die in diesem Kontext entstehenden Rechtsfragen und gibt Antworten, zeigt aber auch auf, in welchen Konstellationen die Kollisionen der Rechtsgüter noch eines öffentlichen und auch wissenschaftlichen Diskurses bedürfen.

Das Präsidium der DGMR bedankt sich bei allen Referenten und Teilnehmern für ihren
Gynäkologe 2014 · 47:72-72

DOI 10.1007/s00129-014-3337-9

(c) Springer-Verlag

Berlin Heidelberg 2014

\section{Infobox Lesetipp}

Weitere interessante Beiträge aus der Zeitschrift MedR Medizinrecht, Ausgabe 09/2013 finden Sie unter dem folgenen Link: http://link.springer.com/journal/350/30/9/page/1

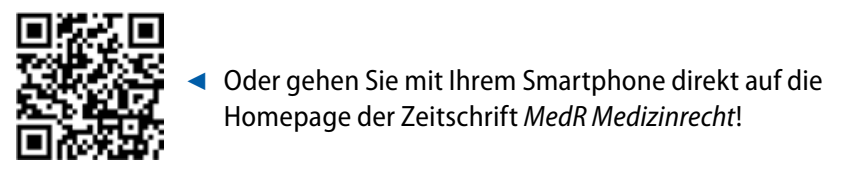

Einsatz und die Beiträge, sowie dem Springer-Verlag, der in bewährter Weise die Drucklegung möglich macht.

Für das Präsidium der DGMR e.V. Rechtsanwalt Dr. A. Wienke

Zusammenfassung aus Wienke A (2013) MedR 31:592-593, DOI: 10.1007/s00350-013-3504-3 